\title{
Análisis de los trabajos presentados en los Congresos Nacionales organizados por la Asociación Mexicana de Mastozoología (AMMAC)
}

\author{
Miguel Briones-Salas ${ }^{1 *}$, Dagoberto Ramos y Yadira Santiago
}

Introduction: The Asociación Mexicana de Mastozoologia was established in 1984 at Mexico City, with four objectives: 1) to study and propose solutions to problems and policies of management and conservation of mammals in Mexico; 2) to gather people interested in mammals in Mexico; 3 ) to promote and organize the interaction between people and groups interested in mammalogy, and 4) to spread and stimulate activities referred to mammalogy through meetings and assemblies, develop curricula and research and organize conferences and congresses about mammalogy. The National Congress of Mammalogy has become the most important academic forum where the progress in analyzed in the nationwide investigation of mammals, held every two years in different locations of the country; brings together about 600 people from different academic levels. It is an event whose purpose is the presentation of papers and discussion. During their activities exchanging ideas and finding solutions to the various problems faced by Mexican mammals. We reviewed and analyzed the content of oral and poster presentations of eleven Mammalogy National Congress organized by the Asociacion Mexicana de Mastozoologia, A. C. (AMMAC) from 1991 to 2012.

Methodology: We reviewed the printed and digital memories of the 11 national conferences held in Mexico mammalogy, from the year 1991 to 2012. Oral and poster presentations for terrestrial, flyers and marine mammals were considered. We organized a database with the content of presentations. We arranged the data base by 11 principal topics and 68 specific topics, as well as other categories including: taxonomic group, states represented and by national and foreign institutions represented in each meeting.

Results: A total of 2,527 oral presentations and posters were registered. The number of presentations since 1991 to 2012 has been increasing over time. The meeting with the higher number presentation was the $X$ NCM organized at the state of Guanajuato in 2010 (476 presentations); following by the XI NCM meeting in the state of Veracruz 2012 (430 presentations). The topics with higher number of studies were ecology $32 \%$ $(n=795)$ and conservation $14 \%(n=356)$, while the least topic included in presentations was physiology $3 \%(n=84)$ and others $1 \%(n=30)$. The state of Oaxaca has been mentioned and studied in $10 \%(n=$ $212)$ of the presentations, while the state of Zacatecas has been the less mentioned .5 \% $(n=11)$ in meeting presentations. The most studied taxa are: Order Carnivora $25 \%(n=657)$, Family Muridae $48 \%(n=346)$, Genus Peromyscus $6 \%(n=233)$ and Specie: Odocoileus virginianus $4 \%(n=170)$. Species in the order Erinaceomorpha were not mentioned at any of the meetings.

Discussion and conclusions: Mammalogy in Mexico has developed significantly in recent years and especially since the founding of the Asociación Mexicana de Mastozoologia. It is demonstrated by the number of works that have been increasing in relation with time. Our revision aims to contribute to guide the future generation of Mexican mammalogists to focus on different regions of Mexico, topics, and taxa for which there is great concern in their study.

Key words: Conferences, conservation, events, history, mammals.

'Laboratorio de Vertebrados Terrestres (Mastozoología), Centro Interdisciplinario de Investigación para el Desarrollo Integral Regional, Unidad Oaxaca (CIIDIR-OAX.), IPN. Hornos 1003, Santa Cruz Xoxocotlán, Oaxaca 71230, México. mbriones@ipn.mx (MB-S), dmendez_11@hotmail.com (DR), honguiyayalandia_12@hotmail.com (YS)

*Corresponding author 
Se presenta un análisis de los trabajos presentados en 11 congresos nacionales de mastozoología (CNM) organizados por la Asociación Mexicana de Mastozoología A.C. (AMMAC), realizados desde 1991 hasta 2012. Los trabajos se organizaron en una base de datos y se agruparon en 11 temas principales y 68 subtemas específicos, grupos taxonómicos, entidades federativas más estudiadas e instituciones nacionales y extranjeras. Se registró un total de 2,527 trabajos, 1,596 en la modalidad de presentación oral y 931 en la modalidad de cartel. El número de trabajos presentados se ha incrementado notablemente a lo largo del tiempo. Los congresos en donde se ha presentado la mayor cantidad de estos son: el X CNM (Guanajuato, 2010) con 476 y el XI CNM (Veracruz, 2012) con 430. Los temas más trabajados fueron sobre ecología $32 \%(n=795)$ y conservación $14 \%(n=356)$, mientras que los menos presentados fueron de fisiología $3 \%(n=84)$. El estado de Oaxaca ha sido el más estudiado $10 \%$ $(n=212)$ mientras que el de Zacatecas ha sido el menos estudiado $0.5 \%(n=11)$. Los grupos taxonómicos más estudiados de acuerdo a cada categoría han sido: el orden Carnivora $25 \%(n=657)$, la familia Muridae $48 \%(n=346)$, el género Peromyscus 6 $\%(n=233)$ y la especie Odocoileus virginianus $4 \%(n=170)$. Destaca la ausencia de estudios sobre el orden Erinaceomorpha. Estos resultados proponen la necesidad de reorientar los estudios en ciertas entidades del país y con temas y grupos taxonómicos poco tratados.

Palabras clave: conferencias, conservación, eventos, historia, mamíferos.

La Asociación Mexicana de Mastozoología (AMMAC), fue fundada en la ciudad de México en marzo de 1984, con cuatro objetivos principales: 1) Estudiar y proponer soluciones conjuntas a problemas y políticas de manejo y conservación de los recursos naturales en México, particularmente de los mamíferos; 2) Reunir a las personas interesadas en mamíferos en México; 3) Promover y organizar la interacción entre personas y grupos interesados en la mastozoología en México; y 4) Difundir y estimular las actividades relacionadas con la mastozoología en México a través de reuniones y asambleas, formular programas de estudio e investigación y organizar conferencias sobre mastozoología.

Desde la fundación de la AMMAC, se han cumplido con el desarrollo de reuniones y conferencias académicas a través de Congresos Nacionales de Mastozoología (CNM). Actualmente, los CNM organizados por la AMMAC tienen como objetivos: a) promover el intercambio de información, ideas y experiencias entre los mastozoólogos profesionistas, estudiantes e interesados en los mamíferos silvestres de México, b) discutir la problemática, y c) proponer alternativas en torno a las necesidades de investigación, conservación y usos sustentables de la mastofauna nacional.

El CNM se ha convertido en el foro académico más importante que permite conocer los últimos avances en la investigación mastozoológica a nivel nacional, se celebra cada dos años en diferentes sedes del país; actualmente agrupa alrededor de 600 personas, principalmente profesionistas y estudiantes de diferentes niveles académicos. Es 
un evento cuya finalidad es la presentación de trabajos y su discusión. Durante sus actividades se favorece el intercambio de ideas, así como la búsqueda de soluciones a los múltiples y diversos problemas que enfrentan los mamíferos mexicanos.

Durante el CNM se mantiene la estructura con presentaciones orales y en cartel, tanto de investigadores como de estudiantes; además de conferencias magistrales presentadas por expertos en las diferentes áreas de la mastozoología, simposios anexos, talleres pre y post congreso, reuniones concurrentes y eventos culturales.

La mastozoología es una ciencia que se ha desarrollado fuertemente durante los últimos 30 años en nuestro país. Destaca el gran número de estudiantes interesados en esa rama del conocimiento científico y el crecimiento de publicaciones por profesionistas mexicanos (Guevara-Chumacero et al. 2001).

La presentación de trabajos de investigación en reuniones académicas (simposios y congresos, principalmente), son de alguna manera un termómetro que indica la directriz de la investigación sobre algún tema o grupo a tratar y son después de las publicaciones un importante medio que indica el camino y desarrollo que se sigue sobre ese tema o grupo a tratar. Guevara-Chumacero et al. (2001), con base en la literatura publicada (revistas científicas, la serie Mammalian Species, capítulos de libros y libros), analizan detalladamente la historia de la mastozoología en México durante 105 años (1890-1995). Entre los resultados destacan a Veracruz como la entidad federativa más estudiada, el $79 \%$ de los trabajos publicados fueron desarrollados principalmente por autores extranjeros y en revistas extranjeras, mientras que los temas más abordados fueron taxonomía y filogenia.

Actualmente no se ha realizado un análisis detallado de los trabajos presentados en los congresos nacionales organizados por la AMMAC. Cada evento por separado ofrece al final un resumen como parte de la información generada durante el congreso pero no lo hace comparativo, ni analiza el grado de avance en el conocimiento de este grupo de animales. Por esta razón, el presente trabajo organiza los estudios presentados durante 11 congresos nacionales de mastozoología desarrollados por la AMMAC, con el fin de analizar las tendencias y desarrollo del conocimiento de la mastozoología en México, considerando los temas tratados, grupos taxonómicos, entidades federativas más estudiadas y la participación de instituciones nacionales y extranjeras. Con este análisis pretendemos orientar a los jóvenes mastozoólogos nacionales a enfocar sus esfuerzos a estudiar los temas, grupos taxonómicos y estados poco abordados en nuestro país.

\section{Métodos}

Se realizó una revisión exhaustiva de las memorias impresas y digitales de los 11 CNM celebrados en México, desde 1991 y hasta 2012. Se consideraron presentaciones orales y de carteles para mamíferos terrestres, voladores y marinos.

La información obtenida en las memorias de los CNM se organizó en una base de datos con la ayuda del programa Access (Microsoft Office 2013 (R). Los campos utilizados fueron: número identificador para cada trabajo relacionado con 11 temas y 69 subtemas (véase Guevara-Chumacero et al. 2001). Los temas principales fueron: alimentación, anatomía y morfología, comportamiento, conservación, distribución, ecología, enfermedades y parasitismo, fisiología, reproducción, taxonomía, filogenia y misceláneos (temas no incluidos en los anteriores). Además se consideró el nombre 
del expositor o expositores, entidad y la institución de adscripción del autor o autores (nacional y extranjero). La institución se subdividió en al menos tres niveles (Institución, dependencia y subdependencia). La mayor parte de los análisis se realizaron tomando en consideración la categoría de institución (nivel más alto). Además se registró el año y número de congreso, la entidad federativa del país donde se desarrolló el estudio (en el caso de ser varias entidades se contabilizó como un trabajo para cada entidad); la ubicación taxonómica para cada especie incluyendo el orden, familia, género y especie de acuerdo al arreglo taxonómico para mamíferos terrestres de Ramírez-Pulido et al. (2005) y de mamíferos terrestres y marinos de Ceballos y Arroyo-Cabrales (2012). Cabe mencionar que se incluye en esta lista todas las especies citadas en los trabajos presentados, incluyendo las introducidas; así mismo, se utilizó la nomenclatura de Ceballos y ArroyoCabrales (2012), pero en muchos de los casos se respetó la nomenclatura indicada por los autores.

Análisis de datos. Se aplicó un logaritmo natural para suavizar los datos al número de trabajos presentados y de instituciones en los 11 congresos analizados, posterior a ello se aplicó una regresión lineal para determinar las tendencias del número de trabajos en el tiempo, con ayuda de los paquetes Excel y Stata V.6.

\section{Resultados}

Un total de 2,527 trabajos han sido presentados en 11 CNM durante el periodo $1991 \mathrm{a}$ 2012, durante los primeros 30 años de vida de la AMMAC (1984-2014). De estos, 1,595 corresponden a presentaciones orales y 932 a presentaciones en cartel. El congreso que mayor cantidad de presentaciones ha tenido fue el X CNM celebrado en la ciudad de Guanajuato (2010) con 476 trabajos, mientras que el evento con menor cantidad de presentaciones fue el I CNM celebrado en la ciudad de Xalapa, Veracruz (1991) con 95 trabajos (Tabla 1). A lo largo de 30 años, el número de trabajos presentados se ha incrementado de manera paulatina $\left(r^{2}=0.82\right)$, aunque se observa un ligero descenso durante los congresos VIII (2006) y IX (2008) para posteriormente llegar a su pico durante el X CNM (2010; Fig. 1).

\begin{tabular}{llllll}
\hline Congreso & Sede & Año & $\begin{array}{l}\text { No.de } \\
\text { trabajos }\end{array}$ & Orales & Cartel \\
\hline I & Xalapa, Veracruz & 1991 & 95 & 72 & 23 \\
II & Guadalajara, Jalisco & 1994 & 107 & 97 & 10 \\
III & Cuernavaca, Morelos & 1996 & 105 & 90 & 15 \\
IV & Xalapa, Veracruz & 1998 & 131 & 96 & 35 \\
V & Mérida, Yucatán & 2000 & 173 & 102 & 71 \\
VI & Oaxaca, Oaxaca & 2002 & 213 & 121 & 91 \\
VII & San Cristóbal de las Casas, Chiapas & 2004 & 292 & 175 & 117 \\
VIII & Zacatecas, Zacatecas & 2006 & 273 & 181 & 93 \\
IX & Guadalajara, Jalisco & 2008 & 232 & 129 & 102 \\
X & Guanajuato, Guanajuato & 2010 & 476 & 285 & 192 \\
XI & Xalapa, Veracruz & 2012 & 430 & 247 & 183 \\
\hline & Promedio & & 230 & 145 & 85 \\
& Total & & 2,527 & 1,595 & 932 \\
\hline
\end{tabular}

Tabla 1. Relación de trabajos presentados en 11 Congresos Nacionales de Mastozoología organizados por la AMMAC (19912012). 
Figura 1. Incremento en el número de trabajos presentados en los 11 Congresos Nacionales de Mastozoología organizados por la AMMAC (1991-2012).
En cuanto a los temas desarrollados para los 11 CNM, el de ecología ha sido el más tratado con $32 \%(n=795)$, seguido de los temas de conservación con $14 \%$ ( $n=356)$, y distribución con $14 \%(n=351)$. Los temas menos tratados han sido sobre fisiología con $3 \%(n=84)$ y el de misceláneos (que incluye subtemas como longevidad, histología, orientación y ecolocación, entre otros más), con tan solo $1 \%$ ( $n=30 ;$ Fig. 2$)$. Al realizar un análisis para cada congreso, sobresale el tema de ecología como el más presentado en cada uno de ellos, seguido del tema de conservación en ocho congresos. El tema de enfermedades se presentó en uno (II CNM) y finalmente el de fisiología en uno (IV CNM; Tabla 2).

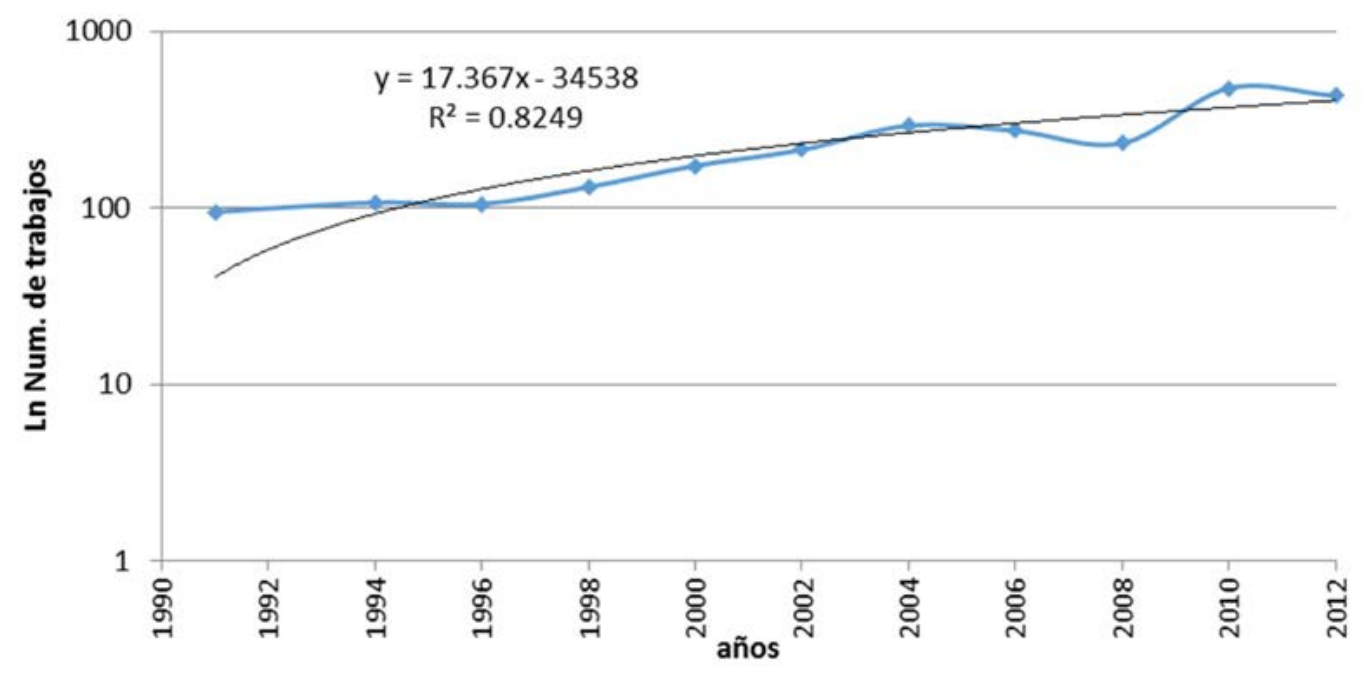

El estado más citado en trabajos presentados fue Oaxaca, con $10 \%(n=212)$, seguido de Baja California (se incluyen las dos entidades: Baja California y Baja California Sur) con $8 \%(n=173)$, Veracruz con $7 \%(n=155)$, Puebla y Jalisco con $7 \%(n=140$, cada uno) y Chiapas con $6 \%(n=126)$. Los estados con menor cantidad de trabajo fueron Aguascalientes con $0.7 \%(n=16)$, Nayarit y Coahuila con $0.6 \%$ ( $n=14$, cada uno), Sinaloa con $0.5 \%(n=12)$ y Zacatecas con $0.5 \%(n=11$; Fig. 3$)$. En 374 trabajos no se menciona el estado donde se realizó el estudio, y en 42 se cita al país (México) como registro general. Solamente en el XI CNM (2012), se presentaron trabajos de los 32 estados mexicanos; en dos congresos (VIII CNM y X CNM) se presentaron trabajos de 31 estados. Mientras que en el I CNM se presentaron trabajos de 23 estados de la República (Fig. 4). Al realizar un análisis de los trabajos presentados en el congreso inicial (I CNM 1991), un congreso intermedio (VI CNM, 2006) y en el último congreso (XI CNM 2012), se observa que al menos nueve estados (Oaxaca, Veracruz, Jalisco, Puebla, Durango, Hidalgo, Chihuahua, Sonora y México), presentan una tendencia de incremento en el número de trabajos presentados entre el primer y último congreso. Se observa también, que seis estados (Guanajuato, Tabasco, Coahuila, Nayarit, Sinaloa y Zacatecas) fueron mencionados por primera vez en trabajos, presentados en el último congreso (CNM 2012; Fig. 5).

En cuanto a autores, la mayoría son de origen mexicano, $97 \%(n=2154)$ y solo $3 \%(n=69)$ fueron extranjeros. De los autores nacionales, la mayoría pertenece a la Universidad Nacional Autónoma de México $21 \%(n=1,604)$, seguido de la Universidad 
Autónoma Metropolitana con $8 \%(n=603)$, el Instituto de Ecología, A. C. con $7 \%$ ( $n$ $=529)$, el Instituto Politécnico Nacional con $7 \%(n=499)$ y la Benemérita Universidad Autónoma de Puebla con $4 \%(n=327)$. Del total de instituciones participantes, 51 de ellas sólo han presentado dos trabajos y 107 de ellas han presentado solamente un trabajo (Tabla 3).

Dentro de las cinco instituciones con mayor número de trabajos presentados se pueden mencionar algunas de sus dependencias con una gran cantidad de trabajos. Destacan de la Universidad Nacional Autónoma de México (UNAM): el Instituto de Biología 31 $\%(n=481)$, el Instituto de Ecología $23 \%(n=353)$ y la Facultad de Ciencias $18 \%$ ( = 270); de la Universidad Autónoma Metropolitana (UAM): la Unidad Iztapalapa 87 \% $(n=519)$, la Unidad Xochimilco $12 \%(n=71)$ y la Unidad Lerma $0.7 \%(n=4)$; del Instituto Politécnico Nacional (IPN): el Centro Interdisciplinario de Investigación para el Desarrollo Integral Regional, Unidad Oaxaca $53 \%(n=257)$, la Escuela Nacional de Ciencias Biológicas $27 \%(n=129)$ y el Centro Interdisciplinario de Investigación para el Desarrollo Integral Regional, Unidad Durango $15 \%(n=74)$; de la Universidad Veracruzana (UV): El Instituto de Investigaciones Biológicas $43 \%(n=119)$, el Instituto de Neuroetología $36 \%(n=99)$ y el Centro de Investigaciones Tropicales $5 \%(n=15)$; finalmente, de la Universidad Autónoma del Estado de Hidalgo (UAEH): el Centro de Investigaciones Biológicas $97 \%(n=228)$.

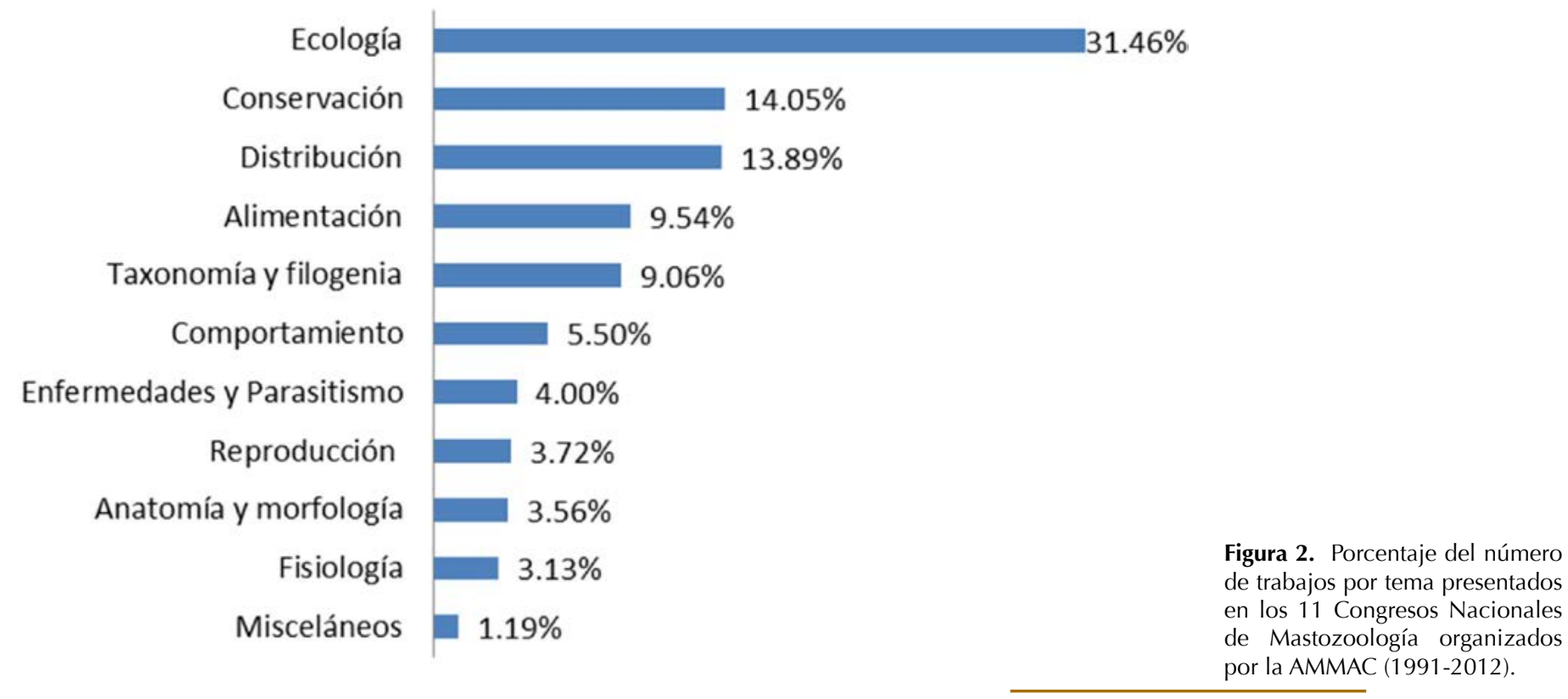

Al realizar un regresión lineal entre el número de trabajos presentados por diferentes instituciones nacionales y el tiempo, se observa una correlación positiva $\left(r^{2}=0.86\right)$; lo que significa que el número de instituciones que presentan trabajos se han ido incrementando conforme ha pasado el tiempo (Fig. 6).

Durante los CNM se han presentado 69 trabajos con datos de mamíferos de 10 países de América. Destacan los trabajos realizados en Estados Unidos con el $29 \%(n=20)$, Colombia $22 \%$ ( $n=15)$, Costa Rica y Guatemala con el $13 \%$ ( $n=9$ cada uno), Ecuador con $6 \%(n=4)$. Entre los países que menos trabajos han presentado son: Brasil, Venezuela y Cuba, $4 \%$ ( $n=3$ cada uno), Argentina con $3 \%(n=2)$ y Chile con $2 \%(n=1)$. 
De acuerdo a los grupos taxonómicos, el orden más citado en trabajos presentados fue Carnivora con $25 \%(n=657)$, seguido de Rodentia con $24 \%(n=644)$, Chiroptera con $19 \%(n=514)$ y Artiodactyla con $12 \%(n=319)$. En un segundo grupo intermedio están los órdenes: Lagomorpha con 6.9\% $(n=157)$, Didelphimorphia con $4 \%(n=$ 107), Primates con $3 \%(n=87)$, Cingulata con $2 \%(n=49)$, Soricomorpha con 1 $\%(n=38)$ y Perissodactyla con $1 \%(n=30)$. Finalmente entre los órdenes menos citados están: Pilosa con $0.9 \%(n=26)$, Cetacea con $0.5 \%(n=15)$, Sirenia con $0.2 \%$ $(n=5)$ y Proboscidea con tan solo $0.04 \%$ ( $n=1$; Cuadro 4$)$. Cabe destacar que no se han presentado trabajos del Orden Erinaceomorpha, de acuerdo al arreglo taxonómico propuesto por Ramírez-Pulido et al. (2005).

Tabla 2. Temas principales presentados en los 11 Congresos Nacionales de Mastozoología organizados por la AMMAC (19912012).

\begin{tabular}{|c|c|c|c|c|c|c|c|c|c|c|c|c|}
\hline $\begin{array}{l}\text { Temas } \\
\text { principales }\end{array}$ & $\begin{array}{c}\text { I } \\
\text { (1991) }\end{array}$ & $\begin{array}{c}\text { II } \\
(1994)\end{array}$ & $\begin{array}{c}\text { III } \\
(1996)\end{array}$ & $\begin{array}{c}\text { IV } \\
\text { (1998) }\end{array}$ & $\begin{array}{c}\text { V } \\
(2000)\end{array}$ & $\begin{array}{c}\mathrm{Vl} \\
(2002)\end{array}$ & $\begin{array}{c}\text { VII } \\
\text { (2004) }\end{array}$ & $\begin{array}{c}\text { VIII } \\
(2006)\end{array}$ & $\begin{array}{c}\text { IX } \\
(2008)\end{array}$ & $\begin{array}{c}X \\
(2010)\end{array}$ & $\begin{array}{c}\text { XI } \\
(2012)\end{array}$ & Total \\
\hline Alimentación & 7 & 6 & 11 & 21 & 29 & 19 & 27 & 29 & 19 & 43 & 46 & 257 \\
\hline $\begin{array}{l}\text { Anatomía y } \\
\text { morfología }\end{array}$ & 6 & 9 & 5 & 5 & 6 & 5 & 8 & 15 & 10 & 12 & 10 & 91 \\
\hline Comportamiento & 6 & 3 & 8 & 4 & 13 & 15 & 20 & 14 & 12 & 15 & 29 & 139 \\
\hline Conservación & 16 & 12 & 15 & 22 & 24 & 27 & 37 & 35 & 33 & 82 & 53 & 356 \\
\hline Distribución & 13 & 17 & 13 & 16 & 19 & 22 & 56 & 32 & 32 & 68 & 63 & 351 \\
\hline Ecología & 32 & 37 & 20 & 37 & 45 & 77 & 90 & 93 & 84 & 145 & 135 & 795 \\
\hline $\begin{array}{l}\text { Enfermedades y } \\
\text { Parasitismo }\end{array}$ & 1 & 0 & 8 & 6 & 7 & 8 & 8 & 11 & 9 & 19 & 24 & 101 \\
\hline Fisiología & 2 & 2 & 2 & 0 & 7 & 8 & 9 & 12 & 8 & 28 & 6 & 84 \\
\hline Misceláneos & 0 & 0 & 3 & 0 & 5 & 2 & 4 & 1 & 3 & 4 & 8 & 30 \\
\hline Reproducción & 7 & 6 & 7 & 10 & 6 & 10 & 11 & 7 & 6 & 14 & 10 & 94 \\
\hline $\begin{array}{l}\text { Taxonomía y } \\
\text { filogenia }\end{array}$ & 5 & 15 & 13 & 10 & 12 & 20 & 22 & 24 & 16 & 46 & 46 & 229 \\
\hline Total & & & & & & & & & & & & 2,527 \\
\hline
\end{tabular}

En cuanto a las familias, las cinco más citadas fueron: Muridae con $12 \%(n=346)$, Phyllostomidae con $10 \%(n=302)$, Felidae con $10 \%(n=286)$, Cervidae con $7 \%$ $(n=214)$ y Canidae con $7 \%(n=209)$. Mientras que las 12 menos citadas fueron: Callitrichidae, Capromyidae, Dasyproctidae, Elephantidae, Equidae, Glyptodontidae, Gomphotheriidae, Hipposideridae, Hominidae, Megatheriidae, Phocoenidae y Rhinolophidae, citadas en solo un trabajo (0.03\%, cada una).

Del total de géneros (202) que habitan territorio Mexicano (Ceballos y ArroyoCabrales 2012), la mayoría de estos (197) se han presentado en al menos un trabajo en alguno de los 11 CNM. Destacan por haberse registrado en una gran cantidad de trabajos: Peromyscus con $6 \%(n=233)$, Odocoileus con $5 \%(n=192)$, Canis con $4 \%$ $(n=145)$, Panthera con $3 \%(n=122)$, Puma con $2 \%(n=97)$, Artibeus y Urocyon con $2 \%$ cada uno ( $n=93)$; por otro lado, 46 géneros se registraron en solo un trabajo $(0.03$ $\%$ para cada uno).

Finalmente, para las especies, se registraron 449 en al menos un trabajo (Apéndice 1), estas incluyen las especies nativas de México. Entre las más estudiadas (> 50 trabajos) se encuentran 12 especies: Odocoileus virginianus con $4 \%(n=170)$, Panthera onca con 3 
$\%(n=122)$, Canis latrans con $3 \%(n=109)$, Puma concolor con $2 \%(n=95)$, Urocyon cinereoargenteus con $2 \%(n=94)$, Artibeus jamaicensis con $2 \%(n=72)$, Leptonycteris curasoae con $2 \%(n=69)$, Pecari tajacu con $2 \%(n=62)$, Nasua narica con $2 \%$ ( $n$ $=60)$, Bassariscus astutus $1 \%(n=58)$, Lynx rufus con $1 \%(n=58)$ y Lontra longicaudis con $1 \%(n=57)$. En un grupo intermedio (entre 10 y 49 trabajos), se registraron 98 especies y en el grupo con el menor número de trabajos $(<10)$, están 339 especies; destacan 155 especies citadas en tan sólo un trabajo.

La mastozoología en México se ha desarrollado de manera importante en los últimos años y mayormente desde la fundación de la AMMAC. Al inicio de la Asociación fueron pocos los jóvenes interesados en esta rama del saber científico. El 16 de marzo de 1984, 14 personas, estudiantes la mayor parte de ellos, firmaron el acta que estableció formalmente la AMMAC; sin embargo, no solo esas 14 personas estuvieron involucradas, aproximadamente 10 más por compromisos sociales y académicos no pudieron estar presentes (F. Cervantes, com. pers.). De 1984 a 2014, han pasado 30 años de proceso y maduración de la AMMAC, prueba de ello es que se han desarrollado 11 CNM que cumplen varios de los objetivos establecidos originalmente por la AMMAC: reunir a todas las personas interesadas en mamíferos en México, y organizar reuniones y conferencias sobre la mastozoología en México.

La mayor parte de los CNM se han desarrollado en las regiones sur o sureste de México: Xalapa, Veracruz (I, IV y XI CNM); Mérida, Yucatán (X CNM); Oaxaca, Oaxaca (VI CNM), y San Cristóbal de las Casas, Chiapas (VII CNM). En la parte central del país se han celebrado cuatro congresos: Guadalajara, Jalisco (II y IX CNM); Cuernavaca, Morelos (III CNM) y Guanajuato, Guanajuato (X CNM). Y en la parte norte solamente uno, (VIII CNM) en la ciudad de Zacatecas, Zacatecas. En este caso, la mayor parte de las instituciones académicas que se enfocan al estudio de los mamíferos en México se encuentran en la región sur y sureste de México y la mayoría de las propuestas para la realización de los congresos han sido planteadas por instituciones de estas regiones del país.

Desde el I CNM en la ciudad de Xalapa Veracruz, se reunieron personas interesadas en el estudio de los mamíferos silvestres (aproximadamente 200), mientras que en el último congreso (XI CNM) se reunieron cerca de 600 personas. La mayor cantidad se presentó en el X CNM celebrado en la ciudad de Guanajuato, Guanajuato, y esto se dio principalmente porque se efectuó en conjunto con el I Congreso Latinoamericano de Mastozoología organizado por la Red Latinoamericana de Mastozoología (RELAM).

Incremento en el número de trabajos presentados. En cuanto a trabajos presentados se observó el mismo patrón, durante el I CNM se presentaron 95 trabajos, a partir de ahí y de manera general el número de trabajos se han ido incrementado con relación al tiempo (Fig. 1), la mayor cantidad de trabajos se presentó durante el X CNM (476), seguramente por el mismo caso arriba mencionado, mientras que en el último (XI CNM) se redujo ligeramente a 430 trabajos. Es evidente que el interés por el conocimiento y conservación de este grupo de animales se incrementa notablemente con el paso del tiempo, principalmente por la asistencia y participación de estudiantes de pre y 
postgrado en ciencias biológicas (obs. pers.). Este mismo patrón se ve reflejado en la producción de artículos científicos, ya que en el lapso entre 1980 y 1989 se publicaron 272 artículos y entre 1990 y 1995 se publicaron 187 artículos (Guevara-Chumacero et al. 2001).

Figura 3. Número de trabajos desarrollados en cada entidad federativa de México presentados en los 11 Congresos Nacionales de Mastozoología organizados por la AMMAC (1991-2012)

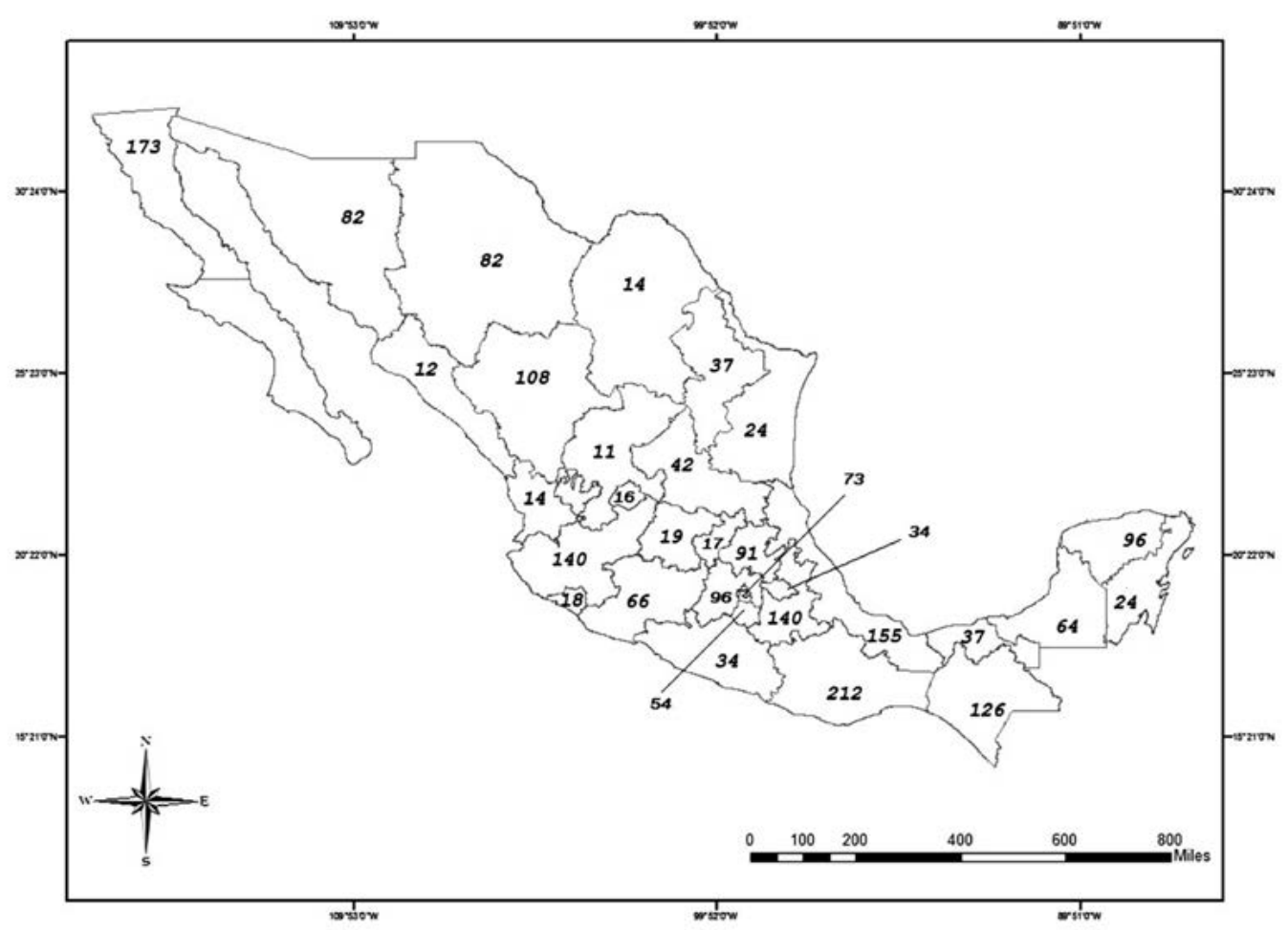

El incremento del número de trabajos presentados se debe seguramente a la formación de muchos estudiantes en los posgrados nacionales e internacionales. Debemos recordar que los socios fundadores de la AMMAC se encontraban en su mayoría estudiando en el extranjero, y a partir de 1985 regresaron a instalarse en varias instituciones nacionales y formar nuevos profesionistas en el área de la mastozoología (F. Cervantes, com. pers.).

Algunos de ellos son los doctores: Alondra Castro (UAM-Iztapalapa), Livia León (Facultad de Ciencias, UNAM), Rodrigo Medellín (Instituto de Ecología, UNAM), Víctor Sánchez-Cordero (Instituto de Biología, UNAM), Juan Pablo Gallo (Centro de Investigación en Alimentación y Desarrollo, A.C., Unidad Guaymas), Héctor Arita (Instituto de Ecología, UNAM), Fernando Cervantes (Instituto de Biología, UNAM) y Gerardo Ceballos (Instituto de Ecología, UNAM), entre otros más. Todos y cada uno de ellos a partir de la década de los 1980’s han formado muchos alumnos que hoy en día son investigadores consolidados en diversos centros y universidades del país.

Temas de los CNM. En cuanto a los temas tratados, el de ecología es el más citado durante los 11 CNM. Un total de 795 trabajos sobre este tema han sido presentados.

Los subtemas relacionados con ecología como estudio del hábitat de diversas especies, estudios de densidad poblacional y estudios demográficos han sido los que más sobresalen. Esta tendencia hacia los estudios sobre ecología tiene una fuerte relación con la formación de los mastozoólogos mexicanos durante la década de los 1980`s, la mayoría de los investigadores anteriormente mencionados realizaron sus posgrados en 
temas afines a la ecología y por consiguiente la formación de profesionales es en esta área. Un factor importante que influye en la inclinación por el estudio de la ecología en México es el refuerzo de instituciones como el Instituto de Ecología, A. C. (creado en 1975), y la creación de instituciones dedicadas a esta área como, el Instituto de Ecología de la UNAM (1988), y el Instituto Nacional de Ecología (INE creado en 1992; Bonilla et al. 1995; véase: http://www.ecologia.unam.mx/organizacion/quienes/historia. htm). En los años de 1990 a 1995 también se presentó un incremento notable en las publicaciones relacionadas al tema de ecología (Guevara-Chumacero et al. 2001).

\begin{tabular}{lrr}
\hline INSTITUCIÓN & Porcentaje \\
\hline Universidad Nacional Autónoma de México & 1,604 & 20.89 \\
Universidad Autónoma Metropolitana & 603 & 7.85 \\
Instituto de Ecología, A. C. & 529 & 6.89 \\
Instituto Politécnico Nacional & 499 & 6.50 \\
Benemérita Universidad Autónoma de Puebla & 327 & 4.26 \\
Universidad Veracruzana & 291 & 3.79 \\
Universidad Autónoma del Estado de Hidalgo & 254 & 3.31 \\
Universidad Autónoma de Yucatán & 233 & 3.03 \\
El Colegio de la Frontera Sur & 204 & 2.66 \\
Universidad Autónoma del Estado de México & 193 & 2.51 \\
Universidad Autónoma del Estado de Morelos & 193 & 2.51 \\
Universidad Autónoma de Baja California & 193 & 2.51 \\
Universidad Michoacana de San Nicolás de Hidalgo & 172 & 2.24 \\
Universidad de Guadalajara & 154 & 2.01 \\
Universidad Autónoma de Querétaro & 152 & 1.98 \\
Centro de Investigaciones Biológicas del Noroeste S.C. & 146 & 1.90 \\
Universidad Juárez Autónoma de Tabasco & 125 & 1.63 \\
Colegio de Postgraduados & 113 & 1.47 \\
Total & 5,985 & 77.94 \\
\hline
\end{tabular}

Otros de los temas más citados en los congresos han sido el de conservación 14 \% $(n=356)$ y el de distribución $14 \%(n=351)$. Estos temas van fuertemente ligados al desarrollo del conocimiento y conservación de las especies de mamíferos mexicanos.

Al incrementarse la lista de investigadores establecidos en diversos estados de la República, muchos de ellos iniciaron sus trabajos recopilando la información disponible de los mamíferos de sus entidades. Algunos caso son para Chiapas (Retana y Lorenzo 2002), Oaxaca (Briones-Salas y Sánchez-Cordero 2004), Baja California (Ríos y ÁlvarezCastañeda 2002) y Veracruz (Gaona et al. 2003), solo por mencionar algunos, además de varios inventarios biológicos regionales y ampliación en la distribución de ciertas especies, muchos de estos trabajos se ven reflejados en los congresos con estudios de zoogeografía $10 \%(n=252)$ y biogeografía en términos generales $3 \%(n=81)$. Sin lugar a dudas, se observó la enorme carencia de información de ciertas especies en cada estado y a partir de ahí surgieron diversos trabajos sobre la conservación a nivel 
estatal, principalmente bajo la categoría de estrategias de conservación de las cuáles se han presentado un gran número de trabajos $3 \%(n=81)$, así como trabajos referentes a colecciones científicas $2 \%(n=60)$ y a áreas naturales protegidas $2 \%(n=57)$.

Figura 4. Número de estados representados en 11 Congresos Nacionales de Mastozoología organizados por la AMMAC, durante los últimos 30 años: 1991-2012

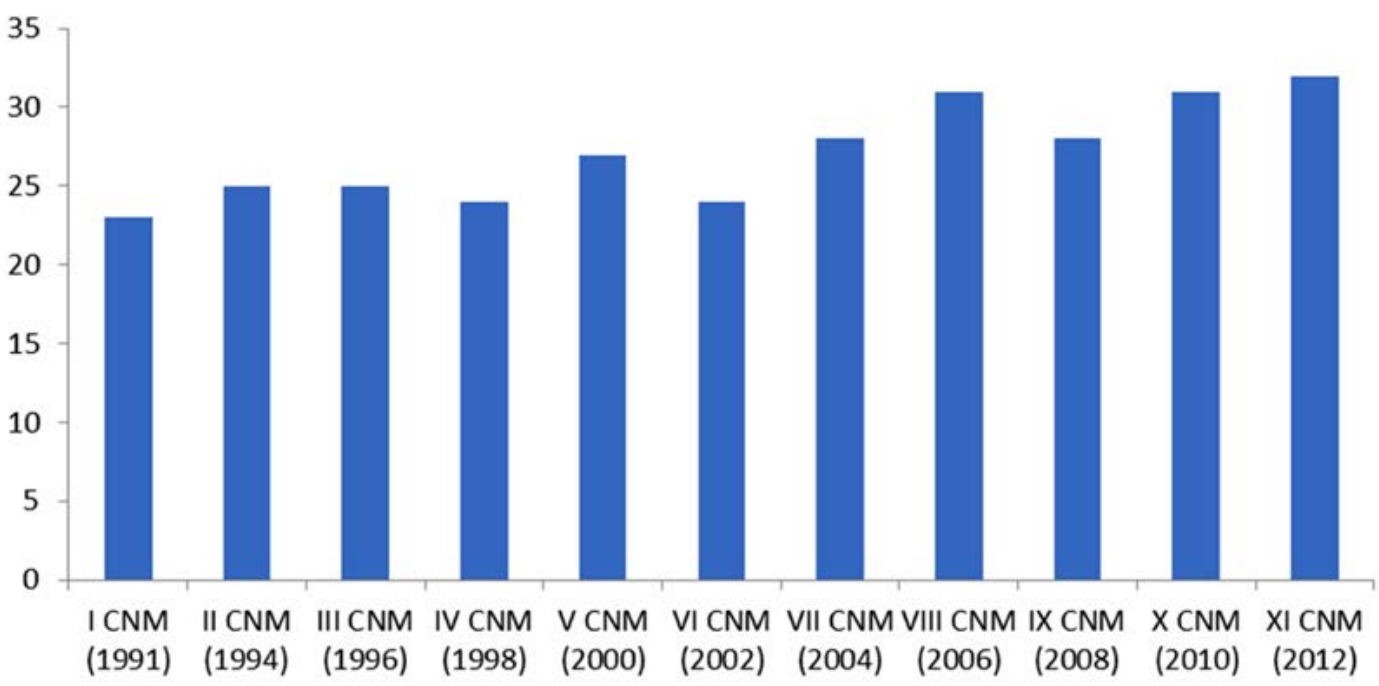

Estados. La mayor parte de los trabajos presentados se han realizado en siete estados de la República (> 100 trabajos): Oaxaca, Baja California, Veracruz, Puebla, Jalisco y Chiapas. Destacan Oaxaca, Veracruz y Chiapas como los estados más diversos del país en cuanto a mamíferos se refiere (Briones-Salas y Sánchez-Cordero 2004; Lorenzo et al. 2008; González 2011). En el caso de Baja California, se incluyen las dos entidades, dado que en muchos de los trabajos no se hacía referencia si pertenecían a Baja California o a Baja California Sur, es por ello que la península es considerada como una sola entidad. Para el caso de Oaxaca, Veracruz y Puebla el número de trabajos se incrementa notablemente en relación al tiempo $\left(r^{2}=0.94 ; r^{2}=0.74\right.$ y $r^{2}=0.88$, respectivamente), esto posiblemente se deba a que el número de investigadores asociados a instituciones locales se incorporaron a ellas a partir del año de 1996, tal es el caso del estado de Oaxaca, Puebla y Chiapas entre otros más. Además de ello, se incrementa la instalación de centros regionales durante los años 1980’s como los Centros Interdisciplinarios de Investigación para el Desarrollo Integral Regional (CIIDIR'es) del Instituto Politécnico Nacional y diversas subdependencias de la Universidad Veracruzana como el Instituto de Investigaciones Biológicas, el Centro de Investigaciones Tropicales y el Instituto de Biotecnología y Ecología Aplicada, entre otras más. En Chiapas, es posible que el número de trabajos sea alto debido a que en el estado existen diversas instituciones, como el Instituto de Historia Natural y Ecología, la Universidad de Ciencias y Artes de Chiapas, el Instituto Nacional de Salud Pública Tapachula, la Universidad Autónoma de Chiapas y diversas Organizaciones no Gubernamentales (ONG's).

Los estados menos citados en trabajos presentados son del norte-centro del país; para Zacatecas solamente se han presentado 11 trabajos; a pesar que el VIII CNM se realizó en la ciudad de Zacatecas durante el año 2006, solamente se presentaron durante el mismo siete trabajos. Posiblemente, se deba esto a que no existían escuelas de Biología o carreras afines que pudieran formar investigadores interesados en la mastozoología. Sinaloa, Nayarit y Coahuila son otros estados con bajos número de presentaciones. En 
términos generales y más recientemente desde 2004 el número de trabajos en ciertos estados de la República ha ido disminuyendo. Una posible causa la representa el ambiente de inseguridad para generar y colectar datos de campo en ciertos estados como Durango, Chihuahua, Tamaulipas y recientemente en Michoacán y Jalisco.

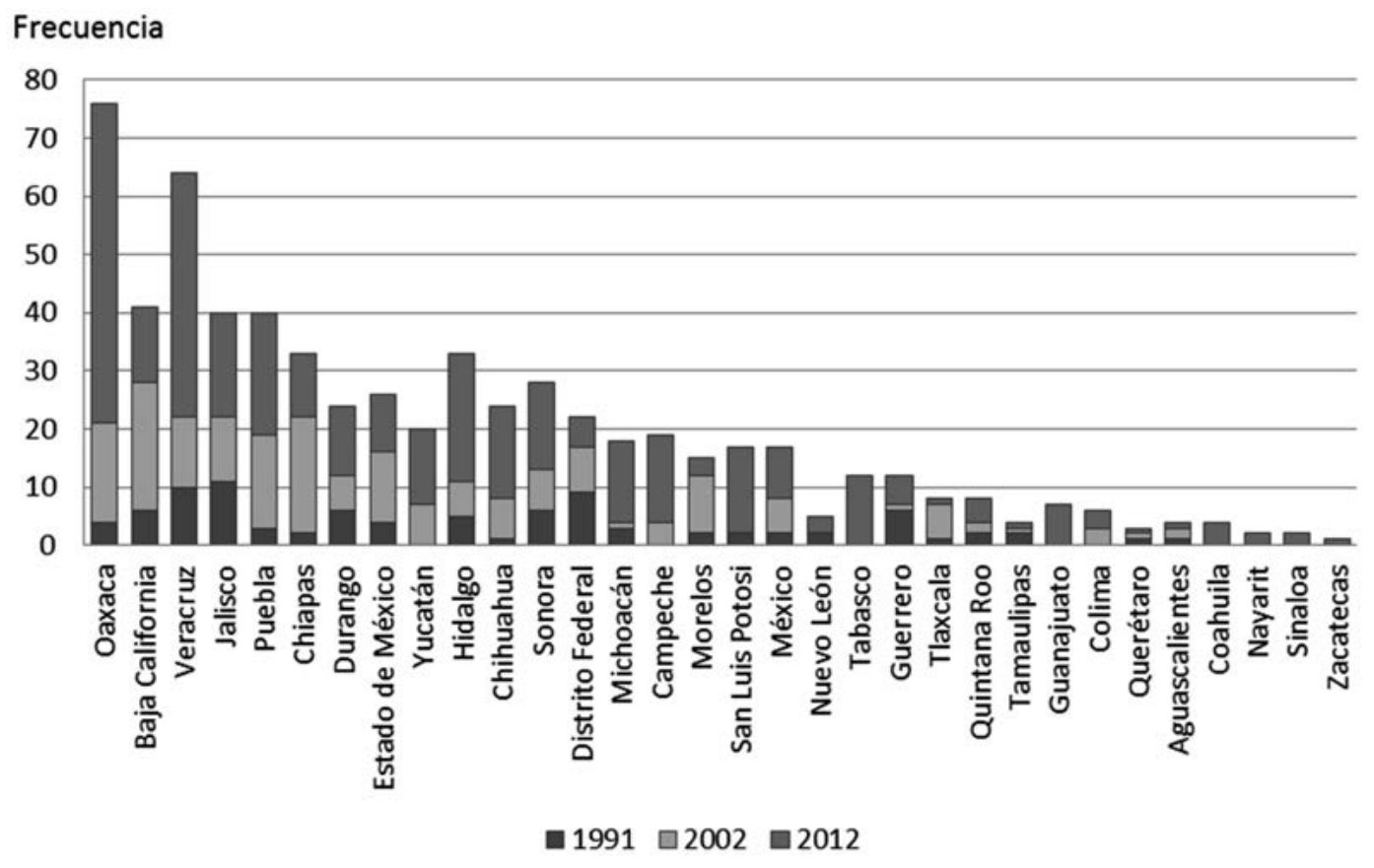

Figura 5. Tendencia del número de trabajos presentados para cada entidad federativa durante el I CNM (1991), el VI CNM (2002) y el XI CNM (2012), organizados por la AMMAC

Instituciones. En cuanto a las instituciones participantes en los CNM, destacan la Universidad Nacional Autónoma de México (UNAM), la Universidad Autónoma Metropolitana (UAM), el Instituto de Ecología, A.C. y el Instituto Politécnico Nacional (IPN), como las cuatro instituciones con mayor cantidad de trabajos durante los CNM. La UNAM se fundó en 1910 y el Instituto de Biología en 1929; con 85 años de historia, sin duda ha sido de las instituciones más antiguas y con mayor trayectoria académica en nuestro país. Cabe resaltar que el Dr. Bernardo Villa "padre de la mastozoología en México" inicia en la UNAM a partir de los años 1940's y fue sin duda el pilar, formador y modelo académico de muchos de los mastozoólogos que formaron la AMMAC.

La UNAM en todos los congresos aparece como la institución con mayor número de participaciones. Otra institución relativamente "antigua" es el IPN, y particularmente la Escuela Nacional de Ciencias Biológicas fundada en 1938, entre sus filas se encontraba el M. en C. Ticul Álvarez, considerado pionero del área en el IPN, formador de excelentes investigadores mexicanos en el campo de la mastozoología. Por su parte la UAM en el Distrito Federal y el Instituto de Ecología, A. C. se fundan en los años 1974 y 1975 respectivamente; estas instituciones contaban desde su formación con profesores mastozoólogos ya consolidados, como es el caso del Dr. José RamírezPulido, que junto con el Dr. B. Villa y el Maestro T. Álvarez han sido los grandes pioneros de la mastozoología mexicana. Esto sin lugar a dudas contribuyó a que los nuevos investigadores de estas instituciones participaran activamente en los CNM. 
Otro factor que pudiera haber influido, es que estas instituciones son de las que mayor presupuesto federal que se les asigna, la UNAM seguido de la UAM y el IPN son las instituciones a las que se le da un mayor monto. El Instituto de Ecología A. C., el cual a pesar de no contar con un gran presupuesto federal por pertenecer al Sistema de Centros Públicos de Investigación (CPI) del Consejo Nacional de Ciencia y Tecnología, se dedica únicamente a la formación de docentes e investigadores lo que lo coloca en el segundo lugar. La ubicación de las instituciones y la matrícula de estas también generan una relación proporcional con el número de aportes ya que de las cuatro instituciones mencionadas con anterioridad la UNAM, la UAM y el IPN son las que cuentan con mayor número de estudiantes y las cuales se encuentran en el Distrito Federal.

Figura 6. Incremento del número de instituciones participantes en los 11 Congresos Nacionales de Mastozoología organizados por la AMMAC, (1991-2012).

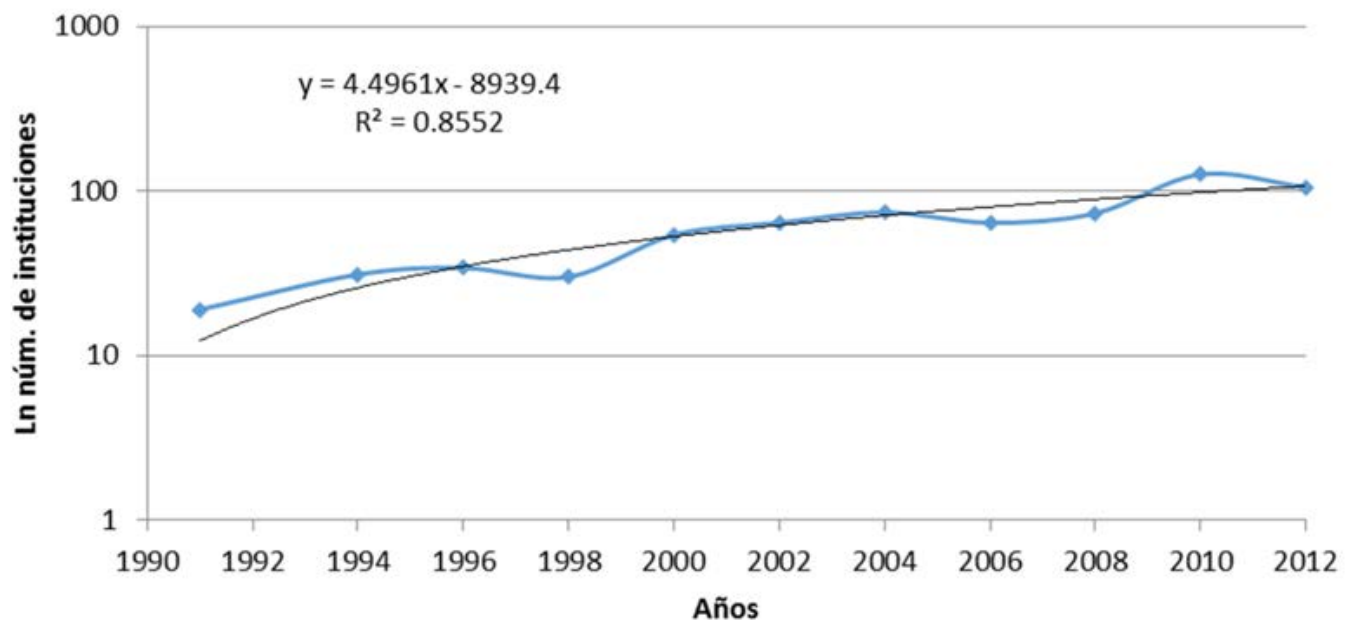

Taxones representados en CNM. Durante los CNM no solo se ha presentado información de especies con distribución actual en México, también se ha contado con la representación de trabajos paleontológicos que enriquecen el conocimiento de especies fósiles en México, ejemplo de esto son los trabajos sobre especies extintas del orden Proboscidea y la familia Equidae. Los trabajos presentados sobre el orden Proboscidea están representados por la familia Gomphotheriidae, con el género Cuvieronius; por la familia Elephantidae con el género Mammuthus, mientras que en Equidae con el género Equus.

Los órdenes mayormente representados son Carnivora, Rodentia y Chiroptera, estos dos últimos están altamente representados por contener un mayor número de especies y ser los grupos con mayor facilidad en su captura. En cuanto al orden Carnivora, éste resulta sumamente atractivo para muchos jóvenes investigadores por ser muchas de sus especies emblemáticas, bandera o carismáticas; también se encuentra ubicado dentro de los primeros lugares por la creciente moda por el estudio de ellos. El crecimiento del estudio de este grupo se ve reflejado a partir del IX CNM en el cual empieza a ocupar el orden más estudiado.

Las familias Muridae, Phyllostomidae y Felidae, fueron de las tres familias con más trabajos presentados, coincide esto con que las dos primeras son también de las familias más numerosas en cuanto a número de especies (135 y 59 respectivamente; RamírezPulido et al. 2005). Dentro de la familia Felidae, se ha trabajado mucho en estas familias 
por contener especies de importancia económica, principalmente los dos grandes felinos depredadores: el jaguar y el puma. Ambas especies han sido estudiadas desde la perspectiva del impacto que tienen sus poblaciones sobre el ganado y recientemente con el impulso del trabajo con trampas cámara, la distribución, poblaciones y ciertos hábitos han sido analizados en muchas regiones de su distribución.

\begin{tabular}{lrr}
\hline Orden & \multicolumn{2}{c}{ Porcentaje } \\
\hline Carnivora & 657 & 24.80 \\
Rodentia & 644 & 24.31 \\
Chiroptera & 514 & 19.40 \\
Artiodactyla & 319 & 12.04 \\
Lagomorpha & 157 & 5.93 \\
Didelphimorphia & 107 & 4.04 \\
Primates & 87 & 3.28 \\
Cingulata & 49 & 1.85 \\
Soricomorpha & 38 & 1.43 \\
Perissodactyla & 30 & 1.13 \\
Pilosa & 26 & 0.98 \\
Cetacea & 15 & 0.57 \\
Sirenia & 5 & 0.19 \\
Proboscidea & 1 & 0.04 \\
Erinaceomorpha & 0 & 0 \\
Total & 2,649 & 100 \\
\hline
\end{tabular}

Tabla 4. Número de trabajos (N), de acuerdo a los órdenes de mamíferos mexicanos presentados en los 11 Congresos Nacionales de Mastozoología organizados por la AMMAC, durante los últimos 30 años (19912012).

Destaca que la especie mayormente trabajada y presentada en congresos sea Odocoileus virginianus, es una especie que ha sido estudiada desde hace muchos años (1850), principalmente por ser parte importante de la alimentación de distintos grupos indígenas y campesino de nuestro país y por tener importancia cinegética (Mandujano 2004). El crecimiento en el número de presentaciones de esta especie coincide también con el número de publicaciones realizadas por mexicanos de 1981 a 2001, con cerca del el 95\% de los estudios (Mandujano 2004). Las otras especies con mayor número de trabajos presentados (> 100 trabajos) fueron: Panthera onca (jaguar) y Canis latrans (coyote). Del primero ya se mencionaron las posibles causas del porque sea una de las especies más estudiadas y, del coyote, quizá por ser una especie de amplia distribución en nuestro país y por tener un gran capacidad de adaptación a medios adversos y estar fuertemente relacionado al hombre (Huxley y Servín 1992).

Del total de especies que se presentan en los congresos (449), 335 corresponden a mamíferos silvestres, mientras que el resto de estas (114), corresponden a mamíferos exóticos, introducidos y extintos o, bien con cambios en su nomenclatura o sinonimia. Resalta que al revisar la lista antes mencionada, no aparecen 210 especies de mamíferos silvestres, de las enlistadas por Ceballos y Arroyo-Cabrales (2012); es decir, el 38.53\% de las especies mexicanas no han sido presentadas en alguno de los CNM. Esto resulta alarmante, porque si bien es cierto que no toda la información llega a los congresos, si es un buen referente de cuantas especies no están siendo estudiadas en nuestro país y 
a las cuales debería ponérseles mayor atención, dado que muchas de ellas son especies raras o bajo algún criterio de conservación.

Resulta necesario que la comunidad científica preocupada por el conocimiento y conservación de los mamíferos mexicanos, considere los resultados aquí presentados y enfoque sus esfuerzos en el estudio de regiones (norte, noroeste y noreste de México), temas (como enfermedades, fisiología e histología) y especies poco tratadas en nuestro país (aproximadamente 155). Observamos también, que el número de trabajos presentados en los 11 CNM, suman más de 2,000; sin embargo muy pocos de estos son publicados en revistas científicas o de divulgación. Esto sin duda, es un problema grave, dado que mucho del conocimiento científico sobre este grupo de animales se queda sólo en la presentación de un congreso y no trasciende a la comunidad interesada y en beneficio de la conservación de los mamíferos mexicanos. Deberán buscarse estrategias para motivar a los estudiantes que presentan sus resultados durante los CNM a publicarlos. La revista Therya, órgano oficial de la AMMAC podría ser la fuente principal para publicar.

Los CNM, han sido sin duda una importante fuente de motivación y generación de mastozoólogos en México. El número de estudiantes interesados ha ido aumentando gradualmente conforme pasan los años y el número de investigadores y profesores instalados en Universidades y Centros de Investigación crece de manera notoria. Hasta hoy en día, la AMMAC ha cumplido cabalmente con uno de los objetivos originalmente planteados. Estamos seguros que el interés sobre el conocimiento y conservación de los mamíferos mexicanos seguirá en crecimiento.

\section{Agradecimientos}

A A. Trujano por la revisión del manuscrito, S. N. Briones y A. G. Monroy por el apoyo en el abstract. MB-S agradece a la Secretaría de Investigación y Posgrado (20130719), a la Comisión de Operación y Fomento a las actividades Académicas (COFFA) y al programa de Estímulos al Desempeño a la Investigación (EDI), las tres del Instituto Politécnico Nacional (IPN) por el apoyo económico recibido, así como al Sistema Nacional de Investigadores (SNI) por su reconocimiento y apoyo. D. Ramos agradece a Conacyt por la beca ofrecida para realizar estudios de posgrado.

\section{Literatura citada}

Asociación Mexicana de Mastozoología. 1991. Memorias I Congreso Nacional de Mastozoología. AMMAC. Xalapa, México.

Asociación Mexicana de Mastozoología. 1994. Memorias II Congreso Nacional de Mastozoología. AMMAC - Secretaría de Agricultura y Recursos Hidráulicos - Universidad de Guadalajara - Subsecretaría Forestal y de Fauna Silvestre. Guadalajara, México.

Asociación Mexicana de Mastozoología. 1996. Ill Congreso Nacional de Mastozoología. AMMAC - Universidad Autónoma del Estado de Morelos. Cuernavaca, México.

Asociación Mexicana de Mastozoología. 1998. Memorias IV Congreso Nacional de Mastozoología. AMMAC -Universidad Veracruzana - Instituto de Ecología, A. C. Xalapa, México.

Asociación Mexicana de Mastozoología. 2000. Memorias V Congreso Nacional de Mastozoología. AMMAC - Universidad Autónoma de Yucatán. Mérida, México. 
Asociación Mexicana de Mastozoología. 2002. Memorias VI Congreso Nacional de Mastozoología. AMMAC - Centro Interdisciplinario de Investigación para el Desarrollo Integral Regional, Unidad Oaxaca, IPN. Oaxaca, México.

Asociación Mexicana de Mastozoología. 2004. Memorias VII Congreso Nacional de Mastozoología "Conservación y Uso Sustentable de los Mamíferos Mexicanos". AMMAC - ECOSUR - Instituto de Historia Natural y Ecología - UNICACH. San Cristóbal de las Casas, México.

Asociación Mexicana de Mastozoología. 2006. Memorias VIII Congreso Nacional de Mastozoología "La Ciencia para la Conservación de los Mamíferos". AMMAC. Zacatecas, México.

Asociación Mexicana de Mastozoología. 2008. Memorias IX Congreso Nacional de Mastozoología "Mamíferos de México: un reto de conservación presente y futuro". AMMAC - Universidad de Guadalajara. Autlán de la Grana, México.

Asociación Mexicana de Mastozoología. 2010. Memorias X Congreso Nacional de Mastozoología y I Congreso Latinoamericano de Mastozoología "Celebrando la biodiversidad de América". AMMAC - Red Latinoamericana de Mastozoología - Dirección de Apoyo a la Investigación y al Posgrado de la Universidad de Guanajuato - Instituto de Ecología del Estado de Guanajuato. Guanajuato, México.

Asociación Mexicana de Mastozoología. 2012. Memorias XI Congreso Nacional de Mastozoología. AMMAC - Universidad Veracruzana. Xalapa, México.

Bonilla, M., R. Herrera, J. L. Rius, y M. José Yacamán. 1995. Sistema de evaluación por pares en los proyectos de investigación y de fortalecimiento de la infraestructura científica y tecnológica. Ciencia y Desarrollo 21:8-23.

Briones-Salas, M., y V. Sánchez-Cordero. 2004. Mamíferos. Pp. 423-447 en Biodiversidad de Oaxaca (García M., A. J., M. J. Ordoñez, y M. Briones-Salas, eds.). Instituto de Biología, UNAM-Fondo Oaxaqueño para la Conservación de la Naturaleza-World Wildlife Fund. Oaxaca, México.

Ceballos, G., y J. Arroyo-Cabrales. 2012. Lista actualizada de los mamíferos de México 2012. Revista Mexicana de Mastozoología Nueva época 2:27-80.

Gaona, S., A. González-Christen, y R. López-Wilchis. 2003. Síntesis del conocimiento de los mamíferos silvestres del estado de Veracruz, México. Revista de la Sociedad Mexicana de Historia Natural 3a . Época 1:91-108.

González, A. 2011. Mamíferos: distribución, endemismo y estado de conservación Pp. In La biodiversidad de Veracruz estudio de estado (Cruz, A, M, Soto, H, Rodríguez, E, Boege, E, Sedas, W, Márquez, M, Primo, G, Castillo, A, Lara, E, Olguín, y C, Landeros, eds.). Comisión Nacional para el Conocimiento y Uso de la Biodiversidad. México. Ciudad de México, México.

Guevara-Chumacero, L. M., R. López-Wilchis, y V. Sánchez-Cordero. 2001. 105 años de investigación mastozoológica en México (1890-1995): una revisión de sus enfoques y tendencias. Acta Zoológica Mexicana (n. s.) 83: 35-72.

Huxley, C., y J. Servín. 1992. De coyotes a coyotes. Ciencias 25:3-8.

Lorenzo, C., E. Espinoza, E. Naranjo, y J. Bolaños. 2008. Mamíferos terrestres de la frontera sur de México. Pp. 147-164 in Avance en el estudio de los Mamíferos de México (Lorenzo, C., E. Espinoza, y J. Ortega, eds.). Publicaciones especiales, 
Vol. II, Asociación Mexicana de Mastozoología, A.C. Ciudad de México, México. Mandujano, S. 2004. Análisis bibliográfico de los estudios de venados en México. Acta Zoológica Mexicana (n. s.) 20: 211-251.

Ramírez-Pulido, J., J. Arroyo-Cabrales, y A. Castro-Campillo. 2005. Estado actual y relación nomenclatural de los mamíferos terrestres de México. Acta Zoológica Mexicana 21:21-82.

Retana, O., y C. Lorenzo. 2002. Lista de los mamíferos terrestres de Chiapas: Endemismo y estado de conservación. Acta Zoológica Mexicana (n. s.) 85:25-49.

Ríos, E., y S. T. Álvarez-Castañeda. 2002. Mamíferos de la Reserva del Valle de los Cirios, Baja California, México. Acta Zoológica Mexicana (n. s.) 86:51-85.

Sometido: 8 de mayo de 2014

Revisado: 1 de agosto de 2014

Aceptado: 22 de agosto de 2014

Editor asociado: Sergio Solari

Diseño gráfico editorial: Gerardo Hernández

\section{Apéndice I}

Lista de especies citadas en los trabajos presentado en los 11 Congresos Nacionales de Mastozoología organizados por la AMMAC (1991-2012). N =Número de trabajos presentados donde aparece(n) la(s) especie(s). Nota: Los nombres científicos se anotan tal cual los autores los mencionan en sus trabajos

\begin{tabular}{llll}
\hline $\mathrm{N}$ & $\%$ & Especies & No sp \\
\hline & & & \\
170 & 4.11 & Odocoileus virginianus & 1 \\
122 & 2.95 & Panthera onca & 1 \\
109 & 2.64 & Canis latrans & 1 \\
95 & 2.30 & Puma concolor & 1 \\
94 & 2.27 & Urocyon cinereoargenteus & 1 \\
72 & 1.74 & Artibeus jamaicensis & 1 \\
\hline
\end{tabular}




\begin{tabular}{|c|c|c|c|}
\hline N & $\%$ & Especies & No sp \\
\hline 69 & 1.67 & Leptonycteris curasoae & 1 \\
\hline 62 & 1.50 & Pecari tajacu & 1 \\
\hline 60 & 1.45 & Nasua narica & 1 \\
\hline 58 & 1.40 & Bassariscus astutus, Lynx rufus & 2 \\
\hline 57 & 1.38 & Lontra longicaudis & 1 \\
\hline 49 & 1.19 & Didelphis virginiana, Leopardus pardalis, Leopardus wiedii & 3 \\
\hline 45 & 1.09 & Procyon lotor & 1 \\
\hline 44 & 1.06 & Sylvilagus floridanus & 1 \\
\hline 43 & 1.04 & Desmodus rotundus & 1 \\
\hline 42 & 1.02 & Sturnira lilium & 1 \\
\hline 41 & 0.99 & Glossophaga soricina, Sigmodon hispidus & 2 \\
\hline 40 & 0.97 & Alouatta palliata & 1 \\
\hline 38 & 0.92 & Ovis canadensis & 1 \\
\hline 37 & 0.90 & Peromyscus difficilis & 1 \\
\hline 36 & 0.87 & Odocoileus hemionus & 1 \\
\hline 34 & 0.82 & Canis lupus, Pteronotus parnelli, Tadarida brasiliensis & 3 \\
\hline 33 & 0.80 & Peromyscus maniculatus & 1 \\
\hline 32 & 0.77 & Dasypus novemcinctus & 1 \\
\hline 31 & 0.75 & Mazama americana & 1 \\
\hline 30 & 0.73 & Tayassu pecari & 1 \\
\hline 29 & 0.70 & Herpailurus yagouaroundi, & 1 \\
\hline 28 & 0.68 & Ateles geoffroyi, Liomys pictus, Mormoops megalophylla, Tapirus bairdii & 4 \\
\hline 27 & 0.65 & $\begin{array}{l}\text { Reithrodontomys megalotis, Liomys irroratus, Ototylomys phyllotis, Peromyscus } \\
\text { melanotis, Sturnira ludovici, rsus americanus }\end{array}$ & 6 \\
\hline 26 & 0.63 & Alouatta pigra, Pteronotus davyi & 2 \\
\hline 25 & 0.61 & Sylvilagus cunicularius & 1 \\
\hline 24 & 0.58 & Artibeus intermedius & 1 \\
\hline 23 & 0.56 & $\begin{array}{l}\text { Artibeus lituratus, Dipodomys merriami, Leptonycteris nivalis, epus californicus, } \\
\text { Mus musculus }\end{array}$ & 5 \\
\hline 22 & 0.53 & Heteromys gaumeri, Peromyscus yucatanicus & 2 \\
\hline 21 & 0.51 & Choeronycteris mexicana, Sciurus aureogaster & 2 \\
\hline 20 & 0.48 & Mephitis macroura, Neotoma albigula & 2 \\
\hline 19 & 0.46 & Eira barbara, Natalus stramineus, Peromyscus mexicanus, Romerolagus diazi & 4 \\
\hline 18 & 0.44 & $\begin{array}{l}\text { Cuniculus paca, Cynomys ludovicianus, Neotoma mexicana, Reithrodontomys } \\
\text { sumichrasti, Tamandua mexicana }\end{array}$ & 5 \\
\hline 17 & 0.41 & Spermophilus variegatus & 1 \\
\hline 16 & 0.39 & Reithrodontomys fulvescens, Spilogale putorius, Taxidea taxus & 3 \\
\hline 15 & 0.36 & $\begin{array}{l}\text { Baiomys musculus, Corynorhinus mexicanus, Lepus flavigularis, Oryzomys } \\
\text { couesi, Perognathus flavus, Spermophilus perotensis }\end{array}$ & 6 \\
\hline 14 & 0.34 & $\begin{array}{l}\text { Anoura geoffroyi, Balantiopteryx plicata, Myotis velifer, Neotomodon alstoni, } \\
\text { Peromyscus boylii, Potos flavus, Sylvilagus audubonii }\end{array}$ & 7 \\
\hline
\end{tabular}


$\mathrm{N} \quad$ Especies $\quad$ No sp

Baiomys taylori, Coendou mexicanus, Dermanura tolteca, Mustela

130.31 frenata, Peromyscus leucopus, Peromyscus melanocarpus, Rattus rattus, Reithrodontomys gracilis, mexicanus, Oryzomys melanotis, Peromyscus furvus, Pteronotus personatus Thomomys umbrinus

Antilocapra americana, Chaetodipus penicillatus, Dermanura azteca Glaucomys volans, Glossophaga morenoi, Lasiurus cinereus, Mazama pandora, eremicus, Peromyscus zarhynchus, Reithrodontomys microdon, Spilogale pygmaea, Vulpes macrotis,

Antrozous pallidus, Artibeus hirsutus, Corynorhinus townsendii, Cynomys mexicanus, Dermanura phaeotis, Diphylla ecaudata, Felis silvestris, Myotis ciliolabrum, Peromyscus gratus, Peromyscus levipes, Peromyscus melanophrys, Peromyscus truei, Philander oposum, Thomomys bottae, Tlacuatzin canescens Spermophilus spilosoma, Agouti paca, Chaetodipus baileyi, Cryptotis mexicana Microtus quasiater, Molossus rufus, Osgoodomys banderanus, Peromyscus perfulvus, Sigmodon leucotis

Canis familiaris, Carollia perspicillata, Carollia sowelli, Cryptotis parva, spicilegus, Reithrodontomys tenuirostris, Tylomys nudicaudus

Arctocephalus townsendi, Carollia brevicauda, Centurio senex, Conepatus mesoleucus, Dasyprocta mexicana, Eptesicus furinalis, Glossophaga commissarisi, Lepus callotis, Marmosa mexicana, Megadontomys nelsoni, Molossus sinaloae, Myotis yumanensis, Noctilio leporinus, Nyctinomops femorosaccus, Nyctinomops macrotis, Onychomys torridus, O. leucogaster, Oryzomys chapmani, Parastrellus hesperus, Peromyscus ochraventer, P. pectoralis, Sciurus aberti, Sigmodon mascotensis, Sorex saussurei, Spermophilus adocetus

Carollia subrufa, Castor canadensis, Choeroniscus godmani, Cervus elaphus, Cryptotis goldmani, C. magna, Dama dama, Dipodomys nelsoni, D. ordii, Eschrichtius robustus, Galictis vittata, Lasiurus ega, Lepus insularis, Myotis volans, Nelsonia neotomodon, Neotoma lepida, N. nelsoni, Notiosorex crawfordi, Orthogeomys hispidus, Peromyscus megalops, P. pseudocrinitus, Trichechus manatus, Tursiops truncates, Xenomys nelsoni

Bison bison, Chaetodipus hispidus, Chiroderma salvini, Cratogeomys merriami, Dasyprocta punctata, Dermanura watsoni, Eumops underwoodi, Glossophaga leachii, Idionycteris phyllotis, Lasiurus xanthinus, Megaptera novaeangliae, Megasorex gigas, Mephitis mephitis, Mimon crenulatum, Musonycteris harrisoni, Myotis fortidens, M. thysanodes, Neotoma bunkeri, Orthogeomys grandis, Peromyscus bullatus, P. caniceps, P. winkelmanni, Procyon pygmaeus, Rattus norvegicus, Sciurus oculatus, Sigmodon alleni, S. ochrognathus, Sorex oreopolus, Spermophilus madrensis, Spilogale gracilis 
Rhogeessa párvula, Balaenoptera physalus, Bassariscus sumichrasti, Callithrix jacchus, Caluromys derbianus, Chironectes minimus, Chrotopterus auritus, Dipodomys deserti, Dipodomys insularis, Erethizon dorsatum, Habromys simulates, Hylonycteris underwoodi, Lampronycteris brachyotis, Lasiurus blossevillii, Lasiurus borealis, Lepus alleni, Macrotus californicus, Marmosa canescens, Megadontomys cryophilus, Microtus oaxacensis, Molossops greenhalli, Mustela nigripes, Myotis auriculus, M. carteri, M. keaysi, Nasua nasua, Noctilio albiventris, Oligoryzomys fulvescens, Oryctolagus, cuniculus, Perognathus amplus, Peromyscus guardia, $P$. guatemalensis, P. mekisturus, $P$. merriami, Promops centralis, Pteronotus gymnonotus, Reithrodontomys bakeri, Reithrodontomys chrysopsis, Reithrodontomys montanus, Reithrodontomys spectabilis, Rhogeessa alleni, R.gracilis, Rhynchonycteris naso, Saccopteryx bilineata, Sciurus deppei, S. niger, Scotinomys teguina, Sigmodon fulviventer, Spermophilus tereticaudus, Tonatia brasiliense, Tylomys bullaris, Ursus arctos, Vampyrum spectrum, Vulpes velox, V. zerda,

Cynomops mexicanus, Ammospermophilus insularis, A. leucurus, Antilope cervicapra, Arctophoca gazella, Artibeus phaeotis, Ateles belzebuth, Balantiopteryx io, Bauerus dubiaquercus, Blarina brevicauda, B. hylophaga, Bocatherium mexicanum, Bos Taurus, Cebus kaapori, Chaetodipus anthonyi, C. fallax, C. siccus, Chiroderma villosum, Chrysocyon brachiurus, Conepatus semistriatus, Cratogeomys neglectus, C. tylorhinus, C. zinseri, Cricetus aureatus, Cryptotis nigrescens, C. obscura, Cuniculus taczanowski, Delphinus delphis, Didelphis albiventris, D. biventris, D. oposum, Dipodomys gravipes, D. spectabilis, Eepycerus melampus, Equus conversidens, E. vulchely, Euderma maculatum, Eumops bonariensis, E. glaucinus, E. perotis, Feresa attenuate, Geomys gophers, Globicephala macrorhynchus, Glyphonycteris sylvestris, Habromys chinanteco, H. ixtlani, H. lepturus, Handleyomys melanotis, Hedyosmum mexicanum, Heteromys australis, H. irroratus, Lepus mellotis, Lontra canadensis, Lophostoma brasiliense, Megadontomys thomasi, Melanomys caliginosus, Mesocricetus auratus, Metachirus nudicaudatus, Micronycteis microtis, M. brachyotis, M. hirsute, M. schmidtorum, Microtus umbrosus, Mimon cozumelae, Molossops termminckii, Molossus ater, M. molossus, Mustela putorios, Myotis milleri, M. nigricans, Nasua nelson, $10.02 \quad$ Natalus mexicanus, Nelsonia goldmani, Neotoma angustapalata, N. anthonyi, N. bryanti, N. goldmani, N. leucodon, N. martinensis, N. micropus, N. varia, Nephelomys albigularis, Nyctinomops aurispinosus, N. laticaudatus, Ondatra zibethicus, Onychomys arenicola, Oryzomys fulvescens, O. palustris, Otonyctomys hatti, Pan troglodytes, Pappogeomys bulleri, P. gymnurus, P. merriami, Perognathus hispidus, P. longimembris, P. maniculatus, Peromuscus furvus, P. beatae, $P$. eva, P. fraterculus, $P$. grandis, $P$. hylocetes, $P$. madrensis, $P$. nasutus, P. pembertoni, P. schmidlyi, P. slevini, Peropteryx macrotis, Phocoena sinus, Phyllostomus discolor, Physeter macrocephalus, Piper hispidum, P. lapathifolium, Procyon insularis, Pseudoca crassidens, Pteronura brasiliensis, Pteropterix macrotis, Reithrodontomys creper, R. hirsutus, $R$. zacatecae, Rhinolophus maclaudi, R. macrotis, R. marsalli, R. paradoxolophus, R. philipensis, R. rex, Rhogeessa tumida, R. mira, Sciurus alleni, S. arizonensis, S. yucatanensis, Sigmodon arizonae, S. toltecus, S. fulviventer, Solanum aphyodendron, S. nigricans, S. schlechtendalianum, Sorex arizonae, S. ornatus, Spermophilus atricapillus, S. beecheyi, Spilogale angustifrons, Stenella attenuate, S.clymene, S. longirostris, Steno bredanensis, Sylvilagus bachmani, S. brasiliensis, S. nuttallii, Tamaulipasaurus morenoi, Tamias bulleri, Trachops cirrhosus, Tylomys tumbalensis, T. watsoni, Vampyressa thyone, Zygogeomys trichopus, 